\title{
Delayed Slipped Capital Femoral Epiphysis After Treatment of Femoral Neck Fracture in Children
}

\author{
Hai Li MD, PhD, Li Zhao MD, PhD, \\ Luyu Huang MD, PhD, Ken N Kuo MD
}

Received: 19 July 2014/Accepted: 14 November 2014/Published online: 27 November 2014

(C) The Association of Bone and Joint Surgeons (B) 2014

\begin{abstract}
Background Slipped capital femoral epiphysis (SCFE) after the treatment of femoral neck fracture is a rare entity in children that poses important treatment challenges.

Case Description We describe the presentation and management of two patients, a 12-year-old girl and a 6year-old girl, each of whom developed SCFE at 5 months and 9 months, respectively, after operative treatment of femoral neck fracture.

Literature Review Five similar cases have been reported in the literature. Along with our two case studies, the

Each author certifies that he, or a member of his immediate family, has no funding or commercial associations (eg, consultancies, stock ownership, equity interest, patent/licensing arrangements, etc) that might pose a conflict of interest in connection with the submitted article.

All ICMJE Conflict of Interest Forms for authors and Clinical Orthopaedics and Related Research ${ }^{\mathbb{R}}$ editors and board members are on file with the publication and can be viewed on request.

Each author certifies that his institution has approved the human protocol for this investigation, that all investigations were conducted in conformity with ethical principles of research, and that informed consent for participation in the study was obtained.

This study was performed in Xin-Hua Hospital affiliated to Shanghai Jiao Tong University School of Medicine, Shanghai, China.
\end{abstract}

H. Li, L. Zhao (ه)

Department of Pediatric Orthopaedics, Xin-Hua Hospital,

Shanghai Jiao Tong University School of Medicine, No. 1665,

Kongjiang Road, Shanghai 200092, China

e-mail: orthzl@126.com

\section{Huang}

Department of Orthopaedic Surgery, Xi-Jing Hospital,

The Fourth Military Medical University, Xi'an, China

K. NKuo

Department of Orthopaedic Surgery, Rush University Medical Center, Chicago, IL, USA average age of all seven patients was 8.9 years (range, 3.612 years; boys, 8.9 years; girls, 9 years). None of the children was in a high weight percentile or had any known endocrinologic disorder. Two had asymptomatic mild SCFE, whereas the others felt unexplained pain with ambulation when the slippage was brought to their attention. Although it is not possible to prove a causal relationship between the initial fracture and the subsequent SCFE, clinical factors such as implant irritation, early return to weightbearing, delayed union or nonunion, coxa vara, and avascular necrosis warrant consideration as potentially related to the subsequent slip.

Clinical Relevance The onset of SCFE after surgical treatment of a femoral neck fracture may reflect inadequate treatment of the fracture. For best practice, we should perform a gentle anatomic reduction, appropriate internal fixation for femoral neck fracture in skeletally immature patients. Postoperative cast immobilization and delayed weightbearing are also important to avoid complication. It is essential for pediatric orthopaedic surgeons to be aware of this clinical scenario to allow prompt recognition and patient treatment.

\section{Introduction}

Slipped capital femoral epiphysis (SCFE) is defined as a posterior and inferior slippage of the proximal femoral epiphysis on the metaphysis of the femoral neck. The exact cause of slippage is uncertain and probably multifactorial, including obesity or increased body mass index, retroversion of the proximal femur, and increased physeal obliquity [4], occasionally associated with endocrine disorders such as hypotestosteronism, hypothyroidism, and hypoestrogenism [14]. However, most SCFE occurs gradually or 
acutely without preceding injury. Some patients recall a minor or nonspecific traumatic event having occurred sometime during the weeks or months before presentation, whereas a few note a specific (and more severe) mechanism of injury such as a fall $[11,16]$. Less commonly, SCFE can occur in patients recovering from a high-energy femoral neck fracture treated with internal fixation.

Femoral neck fractures are rare in the pediatric population and usually result from high-energy trauma [18]. These injuries are extremely challenging to manage because of the high rate of associated complications such as avascular necrosis (AVN), nonunion of the fracture, coxa vara, and premature physeal closure $[2,18,23]$. Most of the complications can be attributed to the local vascular anatomy of the pediatric hip, where there is an absence of vascular anastomosis across the open physis $[3,10]$. The occurrence of delayed SCFE after treatment of femoral neck fracture is another serious complication, although less common. To our knowledge, only five cases of delayed SCFE in the pediatric population have been reported in the literature $[5,8,9,15,21]$.

The diagnosis of SCFE mandates treatment as soon as possible to prevent slip progression that could result in more severe damage to the hip. Delay in diagnosis has been associated with a worse prognosis [12]; therefore, it is critical for pediatric orthopaedic surgeons to be aware of the possible consequence of SCFE when treating femoral neck fractures in children. With this purpose, we are reporting on two new cases along with a review of the literature.

\section{Case Descriptions}

\section{Case 1}

A 12-year-old girl sustained an acute displaced fracture to the base of the femoral neck (Delbet Type III) of her right hip in a traffic accident (Fig. 1A). She underwent a closed reduction by the Whitman method as described by Herring [6] and percutaneous fixation with two partially threaded cancellous screw (Fig. 1B). She started ambulation with partial weightbearing after cast removal 3 months later. However, the radiograph at 5-month followup revealed moderate slippage of the capital femoral epiphysis (Fig. 1 C). Treatment then included simultaneous screw removal, closed reduction by gentle traction with internal rotation, and fixation of SCFE with three Steinmann pins as described by Nonweiler et al. [19] (Fig. 1D). Subsequently, the patient developed AVN of the femoral head and coxa vara (Fig. 1E). A subtrochanteric valgus osteotomy (Fig. 1F) was performed to correct the varus deformity. One year later, she had close to normal ROM in her right hip, but had developed a leg length discrepancy of $2 \mathrm{~cm}$.
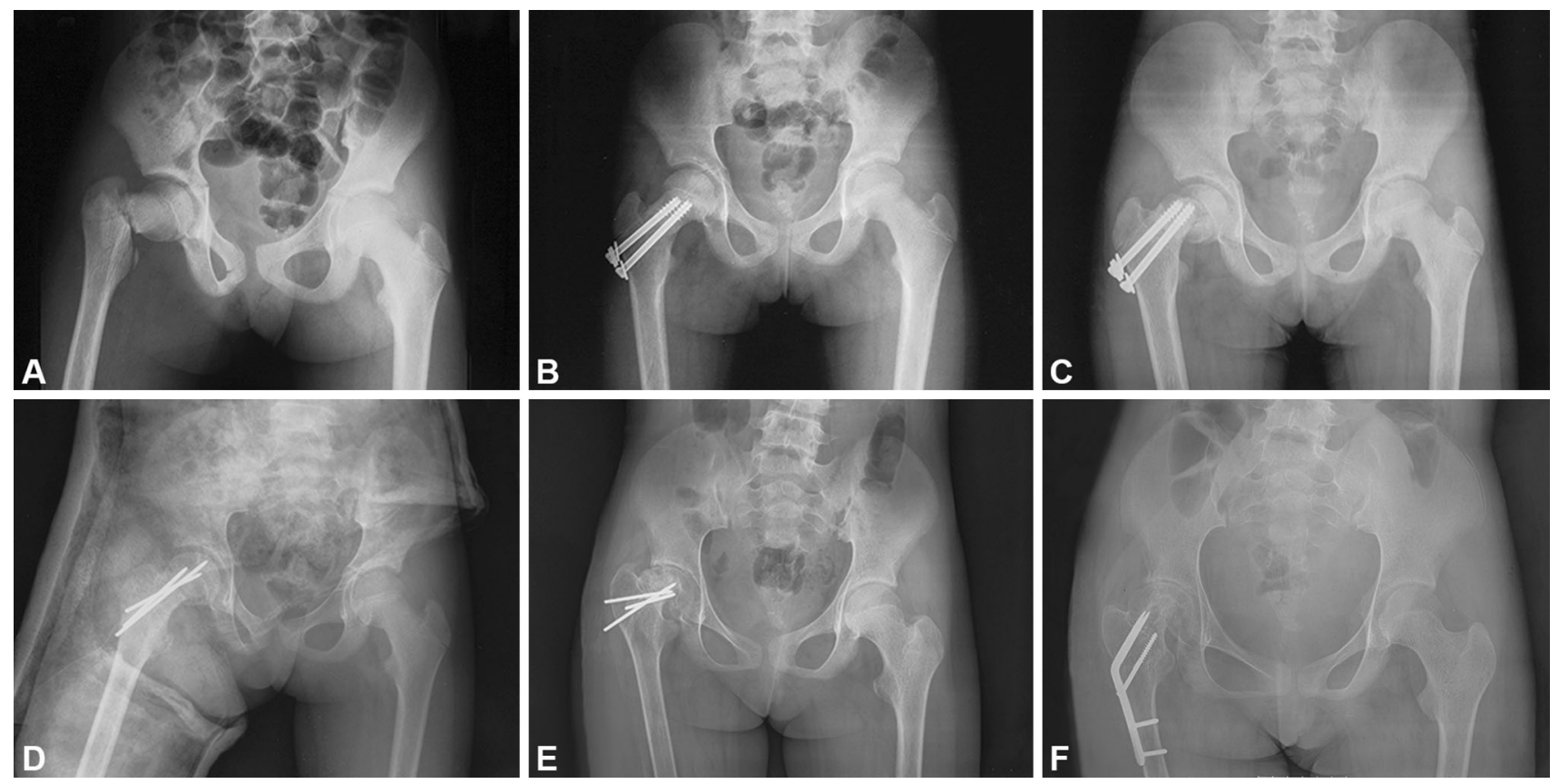

Fig. 1A-F A 12-year-old girl sustained (A) a displaced basal fracture in her right femoral neck. She underwent $(\mathbf{B})$ reduction and fixation with two cancellous screws. (C) SCFE resulted in her right hip at 5-month postoperative followup. (D) After reduction and fixation with three Steinmann pins, the (E) followup radiograph showed AVN of the capital femoral epiphysis and coxa vara. (F) A subchrocanteric valgus osteotomy was performed. 

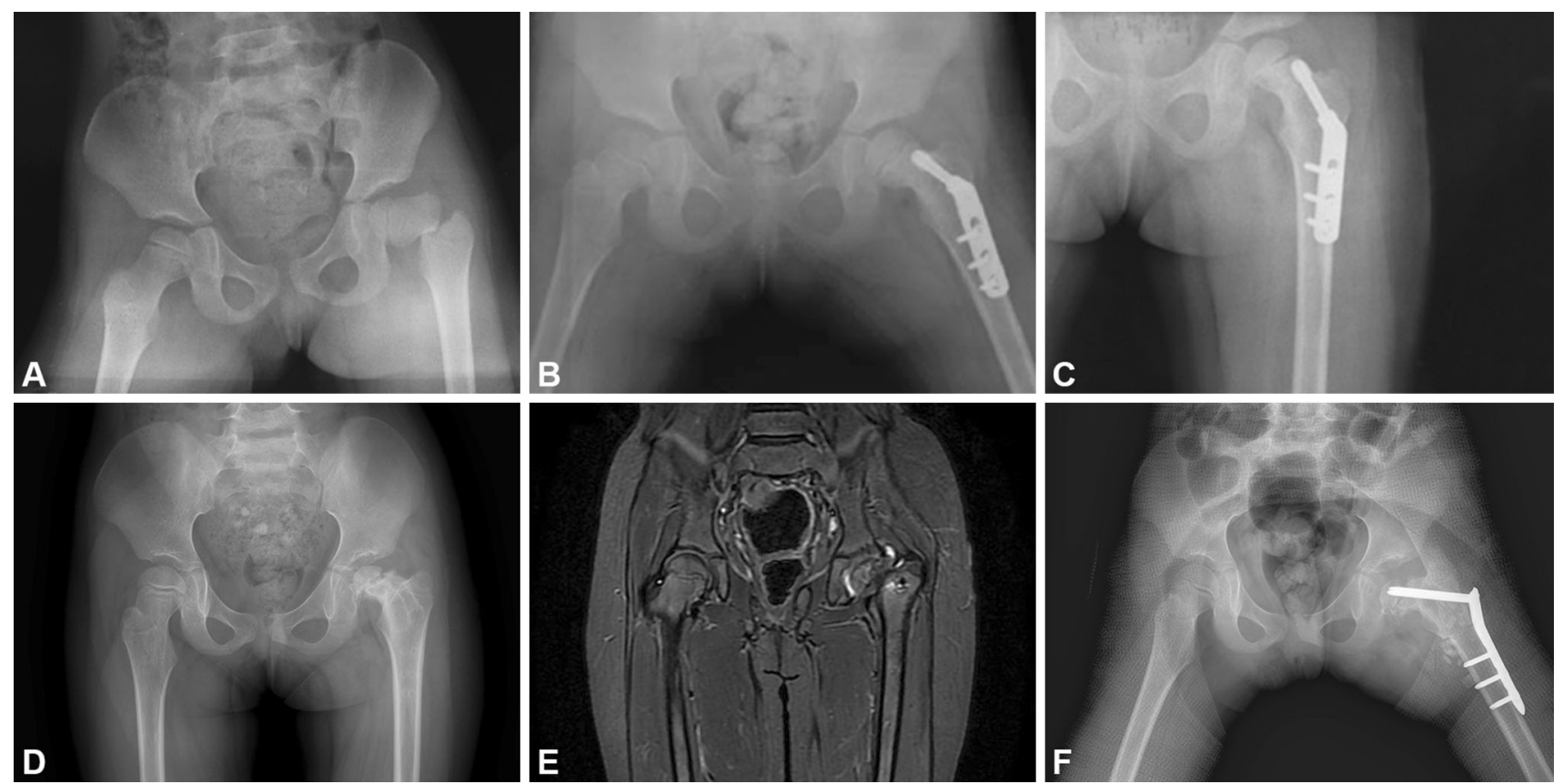

Fig. 2A-F A 6-year-old girl sustained a displaced transcervical fracture (A) in her left femoral neck. She underwent $(\mathbf{B})$ reduction and fixation with a hip compression screw. The tip of the screw was found to be very close to the superior margin of the femoral neck in the postoperative radiograph (C). The hip compression screw was

\section{Case 2}

A 6-year-old girl sustained a displaced transcervical fracture (Delbet Type II) in her left femoral neck after a fall from a height (Fig. 2A). She underwent closed reduction and fixation with hip compression device in other hospital (Fig. 2B-C) and remained nonweightbearing for 8 weeks postoperatively. The patient had nearly full and pain-free range of flexion, extension, internal and external rotation as well as abduction in her left hip postoperatively at that time. Six months later, the hip compression screw was removed. In the next month, she felt unexplained pain in her left hip while ambulating. Radiograph and MR images showed mild slip of the left capital femoral epiphysis with AVN of the femoral head and varus deformity (Fig. 2D-E). An in situ fixation along with subtrochanteric valgus osteotomy was performed (Fig. 2F). At 21-month followup, the patient had residual coxa vara with some limited abduction of left hip without pain.

\section{Literature Review}

We reviewed the reports of five other patients who developed SCFE after surgical treatment of a femoral neck fracture $[5,8,9,15,21]$ (Table 1 ); to our knowledge, these removed 8 months postoperatively. (D) At the 9-month followup, a radiograph (D) showed mild slip with varus deformity. MRI (E) revealed AVN of the capital epiphysis. (F) Postoperative in situ fixation and subtrochanteric valgus osteotomy of the left hip were performed.

represent all of the published examples of SCFE after a surgically treated proximal femoral fracture. Along with our two patients, mean age of all seven patients was 8.9 years (range, 3.6-12 years; boys, 8.9 years; girls, 9 years). Classification of femoral neck fractures were Delbet Type II (transcervical) in four patients and Type III (basicervical) in three patients. The average interval from initial fracture to onset of SCFE was 9 months (range, 5 weeks to 15 months). The left hip was involved in five patients and the right hip in two patients. The degree of slippage was described as mild in five patients and moderate in two patients. None of the children was noted to be in a high weight percentile or had any known endocrinologic disorder. Two had asymptomatic mild SCFE, whereas the others felt unexplained pain with ambulation when the slippage was brought to their attention.

It is impossible to know with certainty whether the delayed SCFE represented a traumatic physeal separation at the time of femoral neck fracture or whether it occurred later. Theoretically, it might be possible for the physeal separation to have remained nondisplaced at the time of injury, failed to heal, and then to displace when the patient resumed walking [22]. However, considering that average time between the onset of slippage and initial trauma was 9.1 months (range, 5 weeks to 15 months), this seems less likely because a nondisplaced physeal separation should 


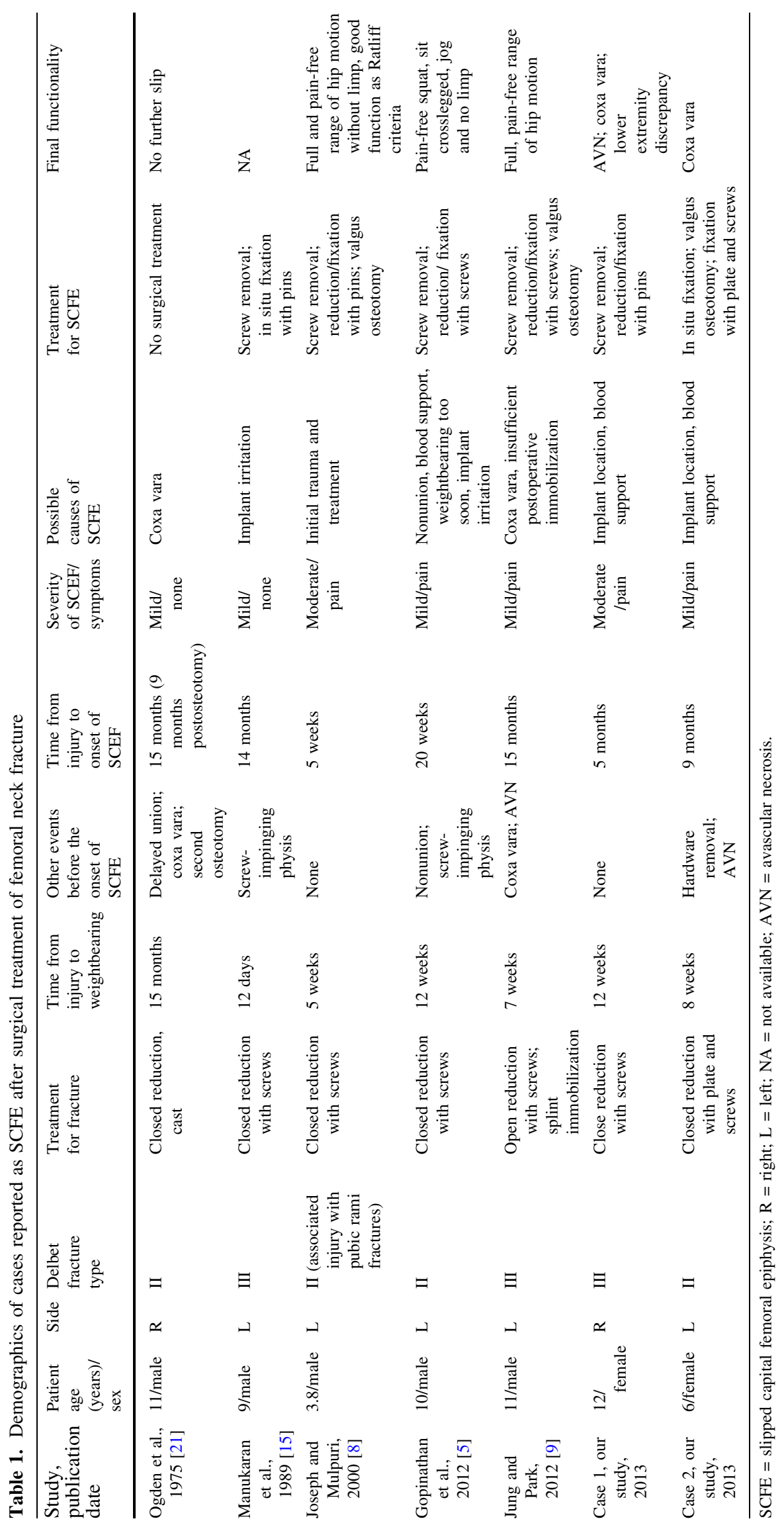


heal in a short time in young children. Because the seven patients in these reports were younger than typical patients with SCFE (boys, 13.5 years; girls, 12 years $[11,13]$ ), it seems possible that the initial femoral neck fracture or subsequent treatment may have in some way contributed to slippage of the capital femoral epiphysis. However, it is difficult to prove a direct causal relationship because most children with femoral neck fracture do not have the complication of SCFE, and only seven cases have been reported to date that we know of, including the two we describe here. Nonetheless, in reviewing the treatment of the seven patients, some associations may plausibly be made, which we discuss in the subsequent section; these associations may include implant irritation, premature initiation of weightbearing, improper postoperative immobilization, delayed union or nonunion, coxa vara, and AVN [5, 8, 9, 15, 21]. One other possible associated factor is excessive retroversion with fixation for the fracture.

\section{Discussion}

Although SCFE after femoral neck fracture in the pediatric population has been reported, an accurate diagnosis may be delayed if the treating physician does not consider late SCFE as an entity in the differential diagnosis of hip pain after surgical treatment of a femoral neck fracture. The cause of SCFE in the setting of recovery from a highenergy hip fracture has not been fully established. Specifically, it is hard to explain why SCFE occurs in younger children, even after the femoral neck fracture has healed. In our study, we report on two pediatric patients with SCFE after healed femoral neck fractures.

Both of our patients also developed AVN in the femoral capital epiphysis after treatment of the fracture (Table 1). AVN of the capital epiphysis is one of the most serious complications that can arise after a femoral neck fracture. Its frequency is variable, ranging from $18 \%$ to $28 \%$ with a higher risk of this complication arising in patients sustaining Delbet Types II and III femoral neck fractures [17, 24]. The high rate of AVN is associated with the anatomy of blood supply for the femoral capital epiphysis and physis, which mainly come from the posterosuperior retinacular vessels [20]. Internal fixation with screws that did not cross the physis was used in both of our patients. Screw placement in one patient was close to the superior corner of the femoral metaphysis (Fig. 1B) and in the other patient was very close to the superior margin of the femoral neck (Fig. 2B-C). Thus, screw placement may compromise blood supply and result in AVN, which might play the role in this uncommon complication, SCFE. Similarly, in two of the patients reported by others [5, 15], the tips of screws were found to be inferior but close to the physis such that they may have impinged on the physis and weakened the interface and perichondral ring between the epiphysis and femoral neck, perhaps leading to the development of slippage of the capital epiphysis. It is important to pay attention to placement of the fixation devices when surgically treating femoral neck fracture in children to avoid serious complications.

Coxa vara is another complication, which occurs in $18.5 \%$ to $30 \%$ of patients after treatment of femoral neck fracture, mainly as a result of inadequate reduction or AVN [7, 24]. Nonunion or delayed union, with an infrequent incidence of $7 \%$ according to one report [7], can be caused by failure to maintain an anatomic reduction such as poor internal fixation, improper postoperative immobilization, and weightbearing started too early [7]. Coxa vara or nonunion in the fracture site increases shearing forces across the growth plate [21], possibly resulting in gradual epiphysis slippage. Three cases reported in the literature had one or more of the complications after fracture treatment [5, 9, 21]. Even in the case with hip spica immobilization for inadequate reduction, the capital epiphysis was subjected to the mechanical disadvantage of the biomechanical environment caused by coxa vara [21]. In another two cases reported in the literature, weightbearing was permitted at less than 6 weeks postoperatively, and both patients developed complications [8, 15]. For basal and transcervical fractures, a hip spica cast is generally recommended for 6 weeks or more in patients with implants not crossing the femoral epiphysis and these patients should not start weightbearing until bone healing is confirmed by radiographs; doing so may increase the risk of nonunion [1].

With regard to treatment for SCFE after femoral neck fracture, all of the patients in the reports we found underwent additional surgery with one exception [21], in which the patient experienced no further slippage. Two patients with mild slippage occurred after treatment with in situ fixation (our Case 2 [15]). Our patient described in Case 2 underwent valgus osteotomy at the time of physeal fixation to minimize shearing forces across the physis. Results of the case from Manukaran et al. [15] where in situ fixation alone with pins was not reported [15]. The other four cases with two mild and two moderate SCFE were treated with reduction, and reduction was maintained with screws or pins after removal of original screws [5, 8, 9]. Two patients underwent subtrochanteric valgus osteotomy at the same time as reduction to minimize shearing forces across the physis and had good results at followup [5, 8]. The patient described as Case 1 in our series was found to have AVN and coxa vara at 5-month followup, which was treated with a third surgical procedure, subtrochanteric valgus osteotomy. Although delayed SCFE after femoral neck fracture was rare, our final results are encouraging. 
In conclusion, pediatric orthopaedic surgeons should be aware of this possible association of AVN and SCFE in the treatment of femoral neck fracture in children. The onset of SCFE may reflect inadequate treatment of the fracture. For best practice, we should perform a gentle anatomic reduction, appropriate internal fixation for femoral neck fracture in skeletally immature patients. Postoperative cast immobilization and delayed weightbearing are also important to avoid complications. It is essential for the treating physician to be aware of this possibility so that recognition and early management can help prevent further complications.

\section{References}

1. Beaty JH, Kasser JR. Rockwood and Wilkins Fractures in Children. Philadelphia, PA, USA: Lippincott Williams \& Wilkins; 2010.

2. Forlin E, Guille JT, Kumar SJ, Rhee KJ. Complications associated with fracture of the neck of the femur in children. J Pediatr Orthop. 1992;12:503-509.

3. Gautier E, Ganz K, Krügel N, Gill T, Ganz R. Anatomy of the medial femoral circumflex artery and its surgical implications. J Bone Joint Surg Br. 2000;82:679-683.

4. Gelberman RH, Cohen MS, Shaw BA, Kasser JR, Griffin PP, Wilkinson RH. The association of femoral retroversion with slipped capital femoral epiphysis. J Bone Joint Surg Am. 1986;68:1000-1007.

5. Gopinathan NR, Chouhan D, Akkina N, Behera P. Case report: bilateral femoral neck fractures in a child and a rare complication of slipped capital epiphysis after internal fixation. Clin Orthop Relat Res. 2012;470:2941-2945.

6. Herring JA. Tachdjian's Pediatric Orthopaedics. 4th ed. Philadelphia, PA, USA: Saunders Elsevier; 2008.

7. Hughes LO, Beaty JH. Fractures of the head and neck of the femur in children. J Bone Joint Surg Am. 1994;76:283-292.

8. Joseph B, Mulpuri K. Delayed separation of the capital femoral epiphysis after an ipsilateral transcervical fracture of the femoral neck. J Orthop Trauma. 2000;14:446-448.
9. Jung ST, Park GH. Slipped capital femoral epiphysis following fracture of the femoral neck: a case report. J Pediatr Orthop B. 2012;21:579-582.

10. Kregor PJ. The effect of femoral neck fractures on femoral head blood flow. Orthopedics. 1996;19:1031-1036.

11. Lehmann CL, Arons RR, Loder RT, Vitale MG. The epidemiology of slipped capital femoral epiphysis: an update. J Pediatr Orthop. 2006;26:286-290.

12. Leunig M, Casillas MM, Hamlet M, Hersche O, Nötzli H, Slongo T, Ganz R. Slipped capital femoral epiphysis: early mechanical damage to the acetabular cartilage by a prominent femoral metaphysis. Acta Orthop Scand. 2000;71:370-375.

13. Loder RT, Aronsson DD, Dobbs MB, Weinstein SL. Slipped capital femoral epiphysis. Instr Course Lect. 2001;50:555-570.

14. Loder RT, Wittenberg B, DeSilva G. Slipped capital femoral epiphysis associated with endocrine disorders. J Pediatr Orthop. 1995;15:349-356.

15. Manukaran MN, Abdul Hamid AK. Slipped capital femoral epiphysis caused by an implant-a case report. Singapore Med J. 1989;30:406-407.

16. Matava MJ, Patton CM, Luhmann S, Gordon JE, Schoenecker PL. Knee pain as the initial symptom of slipped capital femoral epiphysis: an analysis of initial presentation and treatment. $J \mathrm{Pe}$ diatr Orthop. 1999;19:455-460.

17. Moon ES, Mehlman CT. Risk factors for avascular necrosis after femoral neck fractures in children: 25 Cincinnati cases and metaanalysis of 360 cases. J Orthop Trauma. 2006;20:323-329.

18. Morsy HA. Complications of fracture of the neck of the femur in children. A long-term follow-up study. Injury. 2001;32:45-51.

19. Nonweiler B, Hoffer M, Weinert C, Rosenfeld S. Percutaneous in situ fixation of slipped capital femoral epiphysis using two threaded Steinmann pins. J Pediatr Orthop. 1996;16:56-60.

20. Ogden JA. Changing patterns of proximal femoral vascularity. J Bone Joint Surg Am. 1974;56:941-950.

21. Ogden JA, Gossling HR, Southwick WO. Slipped capital femoral epiphysis following ipsilateral femoral fracture. Clin Orthop Relat Res. 1975;110:167-170.

22. Ratliff AH. Traumatic separation of the upper femoral epiphysis in young children. J Bone Joint Surg Br. 1968;50:757-770.

23. Ratliff AH. Fractures of the neck of the femur in children. Orthop Clin North Am. 1974;5:903-924.

24. Yeranosian M, Horneff JG, Baldwin K, Hosalkar HS. Factors affecting the outcome of fractures of the femoral neck in children and adolescents: a systematic review. Bone Joint J. 2013;95:135-142. 